\title{
Geometric modeling design and vibration modal analysis of wind turbine generator blade
}

\author{
Zhiping Zhang ${ }^{1, a}$, Hanwu Liu ${ }^{2, b}$, Liupo Chen ${ }^{2, c}$, Liran $\mathrm{Xu}^{2, \mathrm{~d}}$ \\ ${ }^{1}$ Sch. of Humanities and Social Sciences, North China Institute of Science and Technology, Sanhe, \\ Hebei, 065201, China \\ ${ }^{2}$ Sch. of Mechanical and Electrical Engineering, North China Institute of Science and Technology, \\ Sanhe, Hebei, 065201, China \\ azhipingzhanghk@sohu.com, bhanwu-liu@sohu.com, ${ }^{\mathrm{c}} 793093563 @ q q . c o m,{ }^{d}$ yjs_xIran@163.com
}

Keywords: Wind turbine blade; Vibration stability; Modal analysis; Numerical simulation.

\begin{abstract}
The wind turbine blade is the most complex part of wind turbine generator system (WTGS).The blade itself will produce flutter and divergence under the joint effect of vibration model and aerodynamic force. That the coupling resonance of tower and wind turbine impeller will be produced, when the natural frequency of blade flutter and the frequency of exciting force are the same, will seriously affect the stability of WTGS. This instability of system caused by the coupling resonance will also react upon the blade so as to appear blade fracture phenomenon. This paper analyzed and calculated the vibration model of the wind turbine blade under working condition with the finite element analysis software ANSYS and worked out the vibration frequency and vibration modes of the first nine orders. The analysis results show that the vibration modes of the blade mainly are waving vibration and shimmy vibration in working condition; the displacement values of shimmy vibration are bigger than the displacement values of waving vibration in the cases of similar frequency; Shimmy vibration is the most important vibration mode; In high-order stage, the vibration mode is the superposition of waving vibration, shimmy vibration and torsional vibration modes which is very complex. The results of the study provide the technical support for the improvement and optimization design of the stability of the whole WTGS.
\end{abstract}

\section{Introduction}

Although China's wind-power industry develops fast, it also has a big gap with the international development level so that the domestic equipment mainly depends on import. At present, most types of the domestic large wind-power generator sets are imported, while only $5 \%$ of them are domestic types. In the aspect of theoretical research of wind-power theory, especially there search for control technology of large-scale WTGS, there is still a big gap with the international advanced level. As the most basis component of wind turbine, blade is an important part for energy conversion. Considering that blade is the core component of WTGS, its shape must meet the need to ensure the wind turbine impeller enough lift force and aerodynamic moment and its structure must ensure enough stiffness, strength and stability of the wind turbine.

The vibration modes of wind turbine blade mainly are waving vibration, shim my vibration, torsional vibration modes and the coupling vibration modes of the three modes under blade operating condition. Amplitude of the wind turbine blade is rather small under normal operating condition. The blade bears tension and pressure, bending and torsional force which caused by waving vibration, torque, centrifugal force and gravity, and produces the corresponding waving vibration, shimmy vibration modes and coupled vibration modes of these two modes, while the influence of the torsional vibration is rather limited. Under normal operating condition, the wind turbine blade will be affected by wind load, gravity, centrifugal force. Aerodynamic force makes blade to bear the bending force and torsional force so as to produce waving vibration, shimmy vibration and torsional vibration modes. So, the vibration modes of the most running wind turbine blades mainly are the coupling vibration modes.

The vibration mode is an essential design factor that must be considered under working 
condition of blade. The natural frequency of the wind turbine blade mustn't within the vibration frequency range of rotated wind turbine impeller. And modal analysis is the common method to research the model vibration characteristics of mechanical structure. We can accurately find out the possible problems of design by modal analysis of the wind turbine blade which affected by airflow load, as far as possible to prevent serious influence caused by vibration on the quality of blade. This paper takes $15 \mathrm{~kW}$ horizontal axis wind turbine blade as the research object, and uses the ANSYS software to research and analyze the natural frequency and vibration modes of the blade.

\section{Geometric modeling design of wind turbine blade based on Pro/E}

Wind turbine blade contains three parts of blade tip, blade area and blade root, while the blade cross sections of each part are different and have a certain torsional angle. So we need to draw multiple cross sections when designing in order to obtain relatively accurate results. Take $15 \mathrm{~kW}$ wind turbine blade as an example to design the structure of blade.

According to the calculation formula of impeller radius:

$$
\mathrm{R}=\sqrt{\frac{2 \mathrm{P}}{\rho \mathrm{v}^{3} \mathrm{C}_{\mathrm{p}} \eta \pi}}
$$

In the formula, the output power of wind turbine $\mathrm{P}=15 \mathrm{~kW}$, the wind speed $\mathrm{v}=11.5 \mathrm{~m} / \mathrm{s}$, the wind-power utilization coefficient $\mathrm{C}_{\mathrm{p}}=0.42$, the electromechanical efficiency of wind turbine $\eta=0.92$,the lengthof $15 \mathrm{~kW}$ wind turbine blade is $3.75 \mathrm{~m}$.

As the operational process and output power of three-blade wind turbines are stable, so the three-blade type wind turbines are commonly used in wind-power farm. According to the actual demands, this paper selects three-blade wind turbine as study object and its tip-speed ratio is $\lambda=6$.

When choosing the type of airfoil, generally choose the airfoil that blade root is relatively thick so as to bear the stress of running blade, and the blade root of airfoil should realize the smooth connection to the circular cross-section; blade tip of airfoil should be relatively thin to satisfy the requirement of aerodynamic performance. Therefore, choose the NACA63-421 airfoil profile as the type of blade root, the NACA64-418 airfoil profile as blade area, the NACA64-415 airfoil profile as blade tip.

In the modeling process of blade, according to the structure of the selected airfoil profile, actual space coordinate transformation and solving are needed for the fourteen cross sections, and calculate the chord length and torsional angle of each section, then import the data of all coordinate points in the Pro/E for geometric modeling.

Specifically, according to the airfoil profiles and data of blade sections, determine chord length and torsional angle of each section in EXCEL at first, and calculate the actual space coordinates of each discrete point of the blade cross-section, and then make the calculation results of each cross section into the corresponding ibl files and import them into the Pro/E to generate the spline of each section automatically, and then get a 3D stereogram with fourteen sections, as shown in Fig. 1 . Take the cross section of blade root as starting point and establish a 3D model of wind turbine blade by using the scanning mixed function, as shown in Fig. 2. 


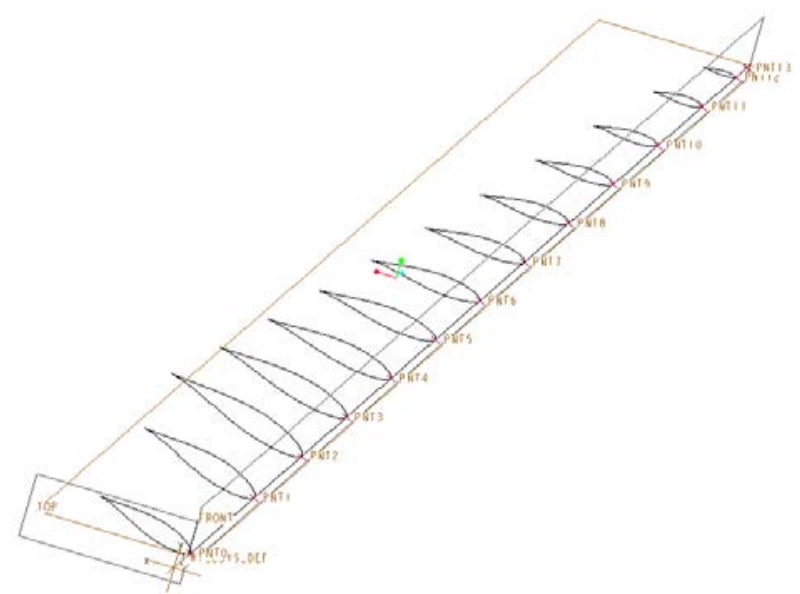

Fig. 1 Cross-section stereogram of blade

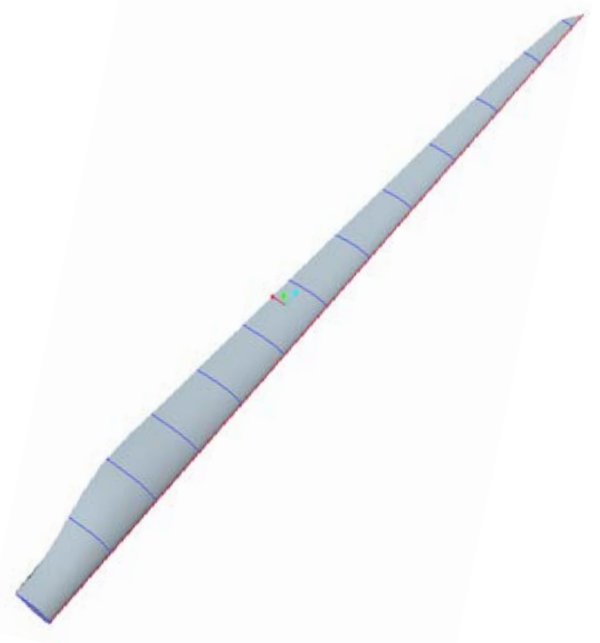

Fig. 2 Model of blade

\section{Modal analysis of the vibration characteristics of blade}

The stress condition of wind turbine blade is the most complex of all parts of wind turbine. Under the mixed effect of vibration mode and aerodynamic force, the blade itself produces flutter and divergence. The coupling resonance will be produced when the natural frequency of blade vibration and the frequency of exciting force are the same so as to seriously affect the stability of WTGS. In order to prevent this situation, the research of natural frequency of wind turbine blade is necessary. In this paper, the modal analysis was carried out on the wind turbine blade by using the finite element analysis software ANSYS in order to optimize the stability of the whole system.

Research of the vibration modals of wind turbine blade mainly is calculating its natural frequency and vibration modes in order to find the reason why blade produces resonance. Blade resonance will produce maximum strain and stress so that the structure of blade can be fatal damaged. The response of blade to the external load is very complex such as waving vibration, shimmy vibration, distortion vibration, and the more complex coupling vibration of these three modes. Apply zero displacement constraint on the blade root in the $\mathrm{X}, \mathrm{Y}, \mathrm{Z}$ direction when analyzing the vibration modes of blade, and then proceed the finite element analysis of the vibrating on modes. The first nine orders vibration frequency is shown in Fig. 3.

According to the above analysis results, draw the conclusions as follows:

(1) Through the contrast of the ninth order vibration mode and the other modes can reveal that the maximum displacement value of the combination vibration of waving vibration, shimmy vibration and torsional vibration is smaller than the maximum displacement value of the combination vibration of waving vibration and shimmy vibration. The low-order vibration modes are mainly waving vibration, shimmy vibration and the coupling vibration of these two modes. The influence of the waving vibration can be clearly seen until the ninth order model diagram, that is, the waving vibration and shimmy vibration are the main vibration of wind turbine blade; 


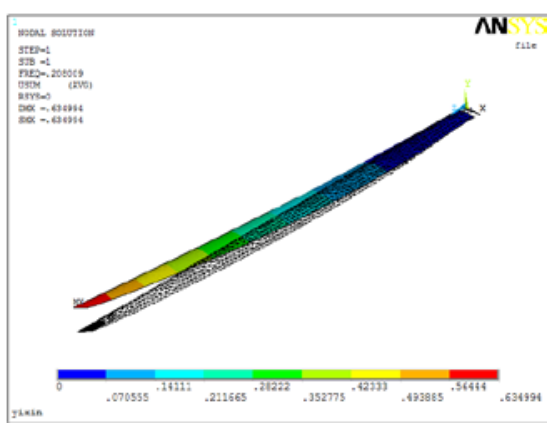

(a) The first order

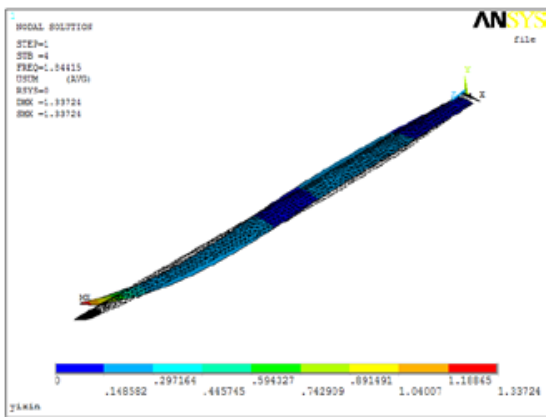

(d) The forth order

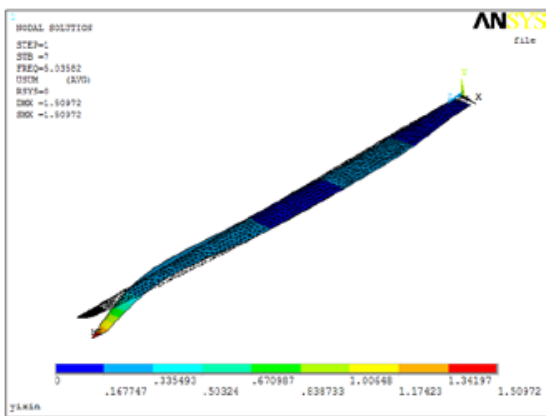

(g) The seventh order

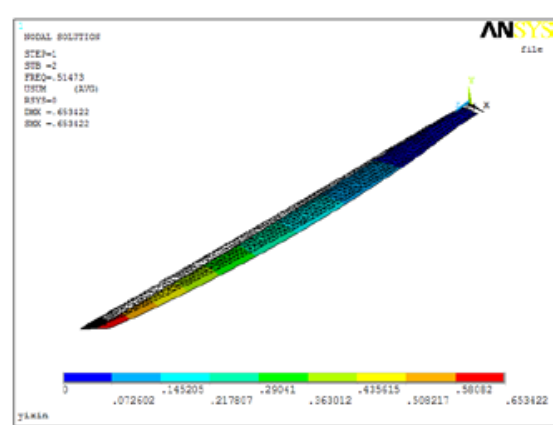

(b)The second order

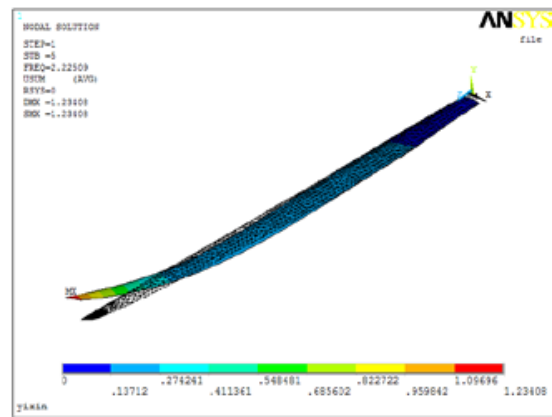

(e) The fifth order

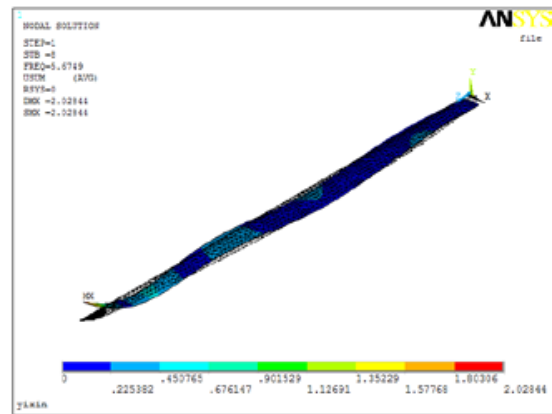

(h)The eighth order

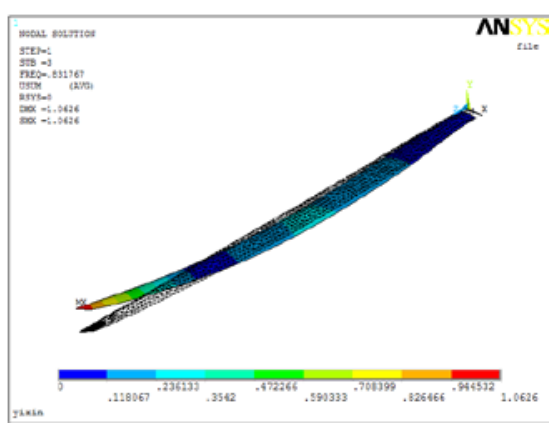

(c)The third order

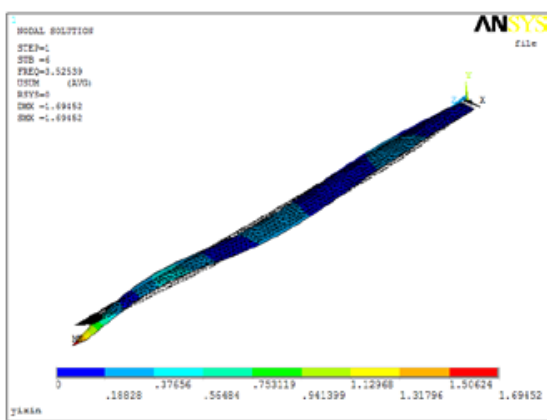

(f) The sixth order

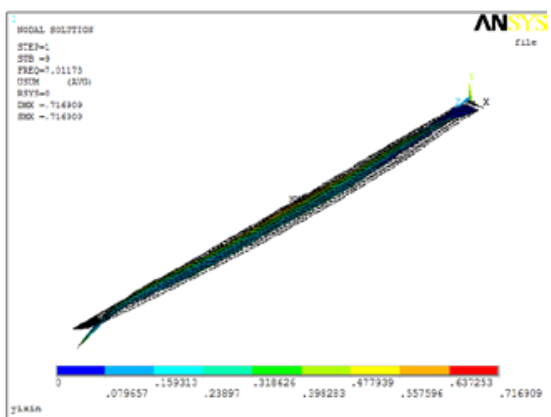

(i)The ninth order

Fig. 3 Results of model analysis

(2) Through the contrast of the sixth order vibration mode and the seventh order vibration mode, the sixth vibration mode mainly is the shimmy vibration mode while the seventh vibration mode mainly is the waving vibration. Although the sixth order modal frequency of $3.52539 \mathrm{~Hz}$ is lower than the seventh order modal frequency of $5.03582 \mathrm{~Hz}$, by contrast, the sixth order model displacement value of the $169.452 \mathrm{~mm}$ is bigger than the seventh order modal displacement value of $150.972 \mathrm{~mm}$. This shows that the influence of the sixth order vibration mode to the blade structure strength is very great.

Table 1 shows the natural frequency of each vibration mode, the maximal displacement value, vibration modes and the explanation of vibration characteristics.

\section{Conclusions}

This paper centers on the project of finite element simulation study of the wind turbine blade under the working condition, and mainly has carried on the research of blade modal analysis and obtains the first nine orders modal vibration modes of $15 \mathrm{~kW}$ wind turbine blade under certain external conditions. The study provides basis theory for the future and further design and development of wind turbine blade in the conditions of rotating, different wind speed or different temperature. Research conclusions are as follows: 
Table 1 Model analysis results and explanation

\begin{tabular}{|c|c|c|c|c|}
\hline Order & $\begin{array}{c}\text { Natural } \\
\text { Frequency/Hz }\end{array}$ & $\begin{array}{c}\text { Maximal } \\
\text { Displacement/mm }\end{array}$ & $\begin{array}{l}\text { Vibration } \\
\text { Mode }\end{array}$ & Vibration Characteristics \\
\hline 1 & 0.208009 & 63.4994 & Shimmy & $\begin{array}{l}\text { Displacement values are gradually } \\
\text { increased from blade root to tip and } \\
\text { the deformation changes smoothly }\end{array}$ \\
\hline 2 & 0.51473 & 65.3422 & Waving & $\begin{array}{l}\text { Deformation changes smoothly from } \\
\text { blade root to tip and overall reflect } \\
\text { strong waving vibration; blade tip } \\
\text { shimmies slightly }\end{array}$ \\
\hline 3 & 0.831767 & 106.26 & Shimmy & $\begin{array}{l}\text { Deformation changes smoothly from } \\
\text { blade root to tip; the tip deformation } \\
\text { is strong; there are two peaks in the } \\
\text { direction of shimmy }\end{array}$ \\
\hline 4 & 1.84415 & 133.724 & Shimmy & $\begin{array}{l}\text { Displacement is wavy change in the } \\
\text { direction of shimmy; reach the } \\
\text { maximum and appear waving } \\
\text { vibration deformation at the blade tip }\end{array}$ \\
\hline 5 & 2.22509 & 123.408 & $\begin{array}{l}\text { Shimmy and } \\
\text { Waving }\end{array}$ & $\begin{array}{l}\text { Displacement changes smoothly near } \\
\text { the blade root; the tip displacement } \\
\text { is biggest; produce the coupling } \\
\text { vibration of shimmy and waving }\end{array}$ \\
\hline 6 & 3.52539 & 169.452 & Shimmy & $\begin{array}{l}\text { Displacement values increase in turn } \\
\text { reduce into wavy from blade root to } \\
\text { tip; blade tip displacement is biggest } \\
\text { and tip produces micro waving } \\
\text { vibration }\end{array}$ \\
\hline 7 & 5.03582 & 150.972 & Waving & $\begin{array}{l}\text { Displacement changes smoothly near } \\
\text { the blade root; blade tip } \\
\text { displacement is the biggest; vibration } \\
\text { mode mainly is waving with a little } \\
\text { shimmy vibration }\end{array}$ \\
\hline 8 & 5.6749 & 202.844 & Shimmy & $\begin{array}{l}\text { Vibration mode mainly is shimmy } \\
\text { with a little deformation produced by } \\
\text { the torsional vibration. }\end{array}$ \\
\hline 9 & 7.01173 & 71.6909 & $\begin{array}{l}\text { Shimmy, } \\
\text { Waving and } \\
\text { Torsion }\end{array}$ & $\begin{array}{l}\text { Combination vibration and complex } \\
\text { vibration model }\end{array}$ \\
\hline
\end{tabular}

1) Accurate solid modeling of wind turbine blade. According to the aerodynamic parameter data of blade and the standard airfoil profiles of NACA, calculate concrete 2D coordinates of each section and complete the 3D coordinate transformation of the 2D coordinates, and then calculate the $3 \mathrm{D}$ coordinates of each point of the blade by using the office software Excel to complete the complex coordinate conversion. Finally, achieve the accurate modeling of the bladewith3D modeling software Pro/E;

2) Modal analysis for wind turbine blade by using the finite element analysis software ANSYS. Mesh the3D entity modeling of wind turbine blade with SOLID45 unit piecewise, carry out the modal analysis with modal extraction method of Block Lanczo, and calculate the first nine orders modals and vibration frequency of the blade under specific conditions. The analysis results show that the waving vibration and shimmy vibration are the main vibration modes of wind turbine blade; in the cases of similar frequency, the displacement values of shimmy vibration are bigger than that of waving vibration, that is, the shimmy vibration is the main vibration modes of all vibration 
modes that affect the blade.

\section{References}

[1] Liping Jiang. Review of wind power development situation at home and abroad of 2008. Economic Power Technologic Economics, 2009, 21 (2):12-14. (In China)

[2] Ahmet Duran, Sahin. Progress and recent trends in wind energy. Progress in Energy and Combustion Science, 2004, 30(5):501-513

[3] J. SelwinRajadurai, T. Christopher, G. Thanigaiyarasu, B. NageswaraRao. Finite element analysis with an improved failure criterion for composite wind turbine blades. ForschIngenieurwes, 2008, 72(3):193-207

[4] Kendall David Arhur. Hinged Blade Model Dynamics for a Horizontal Axis Wind Turbine. The Doctoral Thesis. University of Massachusetts Amherst, 2003

[5] Lida Zhang, Lachun Ren, Rongsheng Chen. Research of shape design and 3D entity modeling of wind turbine blade. Journal of Solar Energy, 2008, 29 (9):1177-1177. (In China)

[6] Tao Yang, Wei Li, Dandan Zhang. Research of shape design and 3D entity modeling of wind turbine blade. Mechanical Design and Manufacturing, 2010(7):190-190. (In China) 